\title{
Aesthetic Origin of Translation Studies
}

\author{
Huani Chen, Qing Zhao, Zihan Liu \\ College of Foreign Languages, Civil Aviation Flight University of China, Guanghan, China \\ Email: chenhuani@cafuc.edu.cn
}

How to cite this paper: Chen, H.N., Zhao, Q. and Liu, Z.H. (2021) Aesthetic Origin of Translation Studies. Open Access Library Journal, 8: e7861.

https://doi.org/10.4236/oalib.1107861

Received: August 18, 2021

Accepted: September 26, 2021

Published: September 29, 2021

Copyright ( $\odot 2021$ by author(s) and Open Access Library Inc.

This work is licensed under the Creative Commons Attribution International License (CC BY 4.0).

http://creativecommons.org/licenses/by/4.0/

\begin{abstract}
This article reviews the development of Chinese and western translation theories. Firstly, it introduces the three stages of the development of Chinese traditional translation theory, namely, from the initial emphasis on "faithfulness" to the emphasis on "literary grace", and then to the later debate on the priority on grace or content. Then, it focuses on the development history of western translation theory from ancient Rome to the $20^{\text {th }}$ century, and summarizes two main periods, namely, the period of rhetoric aesthetics and the period of historical comparative linguistics. The former period began with Cicero, who was deeply influenced by Plato, and in turn influenced the later Bible translation and literary translation; the latter period was divided into two stages: before 1950s, it was mainly influenced by structuralist linguistics, and after 1950s, it was profoundly influenced by emerging language schools and modern ideological trends. Through these introductions, it presents the macro framework of the development history of Chinese and western translation theories.
\end{abstract}

\section{Subject Areas}

Linguistics

\section{Keywords}

Translation Theory, Translation History, Translation Views, Linguistics

\section{1. 引言}

语言学是近代译学思想的主要源泉。随着翻译研究和实践的深入, 语言 学视角逐渐呈现出其局限性。因此, 有必要对译学曾经的思想渊源一一美学 进行回顾, 汲取营养, 以弥补语言学视角的不足。目前对这个问题进行研究 的学术成果有董务刚的《美学与翻译研究》和刘宓庆的《翻译美学导论》。 本文就此展开的探讨将为该领域进一步研究提供线索。 


\section{2. 古典翻译思想}

\section{1. 中国古典翻译思想}

大约于 1900 多年前开始的佛经翻译是中国传统译论的先声。作为一项新 事物, 没有什么系统的译论可以作为指导。为更好地进行翻译工作, 翻译者 只能从先哲之言中寻找依据, 因此与哲学、美学有着天然的联系。译者只好 从本土典籍中获取相关的灵感, 于是, 《道德经》 “信言不美, 美言不信” 成了当时对翻译活动影响最大、最受译者重视的原则一一真实的话往往不美 妙动听, 而美妙动听的言辞往往不真实, 不可信。这句话反映了老子对 “美” 的基本态度一一重信、重实, 对虚饰之美基本采取一种否定的态度。因此, 起初的佛经翻译都很朴实, 佛经的译者和持诵者均以 “信” 为准则。比如, 竺法护的译经便文辞古朴。其后, 随着佛教的普及和在贵族、士大夫阶层中 影响力的不断提升, 对译本的文学性、可读性提出了越来越高的要求, 于是, 文采受到格外关注。然而物极必反, 过分强调 “文采” 又导致以辞害意甚至 削足适履。比如, 支谦的译文便过于雕琭。围绕两种翻译策略各自的优势与 不足, 后世在译论领域产生了 “文” “质”之争。鸠摩罗什弟子僧肇认为竺 法护和支谦都有失偏颇, 而主张一种兼顾的方式。但鸠摩罗什本人的翻译一 般仍被视作是文派。受中庸思想的影响, 中国古代先哲基本持一种调和的态 度。文质之争最终以慧远的文质并重思想结束, 后世佛经译者虽仍有偏文和 偏质的代表, 也主要是因为译者个人的风格和偏好有所不同。在思想认识上, 兼顾文质是译者们共尊的原则 [1]。荀子 “美善相乐” 也体现了这一精神。刘 劦思则基本主张质主导下二者的相辅相成[2]。

\section{2. 西方古典翻译思想}

同中国译学的肇始情况相似, 西方译学最初也没有系统的理论, 而是从 直接哲学和美学宝库中汲取营养。这个过程持续了 1900 年左右, 即从公元前 的西塞罗(Marcus Tullius Cicero, 106-43BC)到 19 世纪的歌德。其间, 译者和 翻译评论者对翻译的论述基本都是以辞章美学为视角的, 代表人物包括西塞 罗、马丁・路德, 以及歌德。西塞罗和稍后的抒情诗人昆塔斯 ・霍拉斯(Quintus Flaccus Horace, 65-8BC) 被共同视为西方译论的奠基人。柏拉图是西塞罗哲学 上的思想源泉, 直接启发了后者对于翻译的看法。柏拉图古典美学观主要体 现在以下几方面:

首先, 将美本身提炼为一个独立范畴。其内涵为使一切美的事物成其为 美的那种关键特质。例如, 玛丽很美, 珍妮也很美, 虽然两人五官差异很大, 但使两人成为美女的那种共同特质可以被提炼出来，好比从金矿里提炼出金 子。言外之意, 存在这样一个 “标准美女” , 而玛丽、珍妮都具备了其部分 特征。翻译是更换表达美的标签, 剥离原来的语言, 用新的语言表达同样的 内容是可能的, 不存在什么不可译的问题。

接着, 指出了美的相对性与美的绝对性的关系。比如, 陶罐的美始终不 及美女的美, 而美女则无法与天神謧美。陶罐的美可以从形状、比例等角度 进行把握, 而把握美女的特征难度要大得多。但如前所述, 这依然是可以提 
炼出来的。天神的美则更加虚无缥纱。因此, 越是高层次的美, 越难通过理 性来认识, 而要靠整体把握和感受。持此观点的译者会更倾向于意译。

再次, 提出美的理念论。“理念” 是柏拉图思想体系中的一个重要范畴, 美本身是绝对的、永恒的。所有美女都是标准美女的一个化身, 现实中的美 女千差万别, 但标准美女形象唯一、永恒不变。不同原作背后也有共同的美 的理念, 再现这种美是译者的使命。

最后, 提出美的认识论。认为对美的把握是一个循序渐进的过程, 须从 感性到理性。具有一定文学素养的人读莎士比亚的诗歌, 起初往往只感觉不 错, 经过文学理论家的分析解读, 会进而从深层次理解她的美。西方译论从 美学传统发展到语言学立场的实际过程也符合这一原则。今天看来, 感性认 识与理性认识可以互补, 建立在理性认识基础上的整体性感受可以激发出新 的灵感，而这是东方思想的特长。

但是, 当进行翻译实践和对翻译活动进行反思和研究时, 西塞罗并未僵 化地运用柏拉图的理念观, 而是对其辩证吸收。他对 “绝对理念论” 提出了 挑战。根据柏拉图的观点, 每个词背后都有一个所谓的绝对理念, 这个绝对 理念又可以直接用另一种语言中的某个词表达, 即 $\mathrm{A}$ 语言中的词可以直接置 换为 $\mathrm{B}$ 语言中表达同一理念的词。理念是根本, 不同语言中的词只不过是 “标 签” ，翻译实际上就是更换标签。这种观点将翻译活动机械化、简单化了。 西塞罗反对这种观点, 遵循意义对意义的翻译准则, 从而突破了字对字翻译 的藩篱。西方译论呈现出一种继承关系。如古希腊思想家泰勒斯 (Thales, 624BC-546BC)美学思想中 “自然气势” 这一范畴同样受到西塞罗的重视, 而 西塞罗又影响了后来的修辞学家昆体良 (Marcus Fabius Quintilianus, c35-c100)。昆体良是一位雄辩大师, 他的这一身份使他对文采和感染力给予 更多的关注，因此他主张意译。昆体良的这一主张又对几乎与他同时代的史 学家李维(Livy, 59BC-17AD) 以及诗人奥维德 (Ovid, 43BC-17AD)起到了示范 作用。物极必反, 昆体良、李维、奥维德等人又过分重视文采, 这种追求藻 饰的风格对后世的翻译也产生了一定的负面影响。古罗马诗人贺瑞斯(Horace, 65BC-8BC) 在一定程度上继承了西塞罗的主张, 他重视并赞赏行文的 “自然 气势”, 以及泰勒斯式的自然美, 但反对过分雕玩。哲学一美学传统的主要 代表为杰罗姆以及奥古斯丁。前者主张意译与直译的折中与统一：一方面强 调 “不能逐词对译”, 认为译文应该根据译入语特点 “表达外语的风格特征”, 并将卖弄学问的死译译者所谓的 “精确” 斥为 “迂腐” 的 “学究气的做法”; 但他又区别对待不同的文本, 主张文学用意译, 而《圣经》则应当直译, 体 现了他对待后者时的格外严谨一一这与他的神学背景有关。总之, 应该 “有时 意译，有时直译”，灵活处理。奥古斯丁则继承发展了亚里士多德和古希腊符 号理论(the theory of signs)。西方译论在奥古斯丁之后进入了一个沉寂期[3]。

\section{3. 中世纪翻译思想}

\section{1.《圣经》翻译思想}

公元 5 世纪中期到 17 世纪，欧洲的科技文化发展处于停滞期，宗教几乎 
成了唯一的精神生活内容, 《圣经》翻译是译者关注的焦点。14 世纪 80 年 代初, 《圣经》的第一个英文译本应运而生, 译者为英国翻译家约翰 - 威克 里夫, 这本《圣经》也被称为《威氏圣经》。威克里夫的翻译目的是使基督 教在英国传播开来, 因此文本一定要易于为广大民众接受, 于是他力求译文 的流畅、易懂。大约 15 世纪初, 柏维(John Purvey, 1354-1428)对《威氏圣经》 进行了修订, 明确提出译者应当遵循 “句对句” 翻译的原则, 而不应单纯拘 泥于词义, 从而使译文如同原文一样清晰流畅。威克里夫和柏维为 18 世纪末 泰特勒的翻译理论构建提供了先例。泰特勒 “三原则” 基本是继承了 “西塞 罗一霍拉斯一杰罗姆” 的意译传统。16 世纪最有影响的《圣经》翻译家是马

丁 - 路德。美学是他翻译观中重要的组成部分和理论源泉。在他看来, 语法 仅仅是厘清语言形态(如动词变化方式、句子结构、词语搭配等)的手段。当 然, 这不是说语法不重要, 而是强调不应使语法结构成为束缚译文的桎梏。 这意味着译者应发挥主观能动性, 不仅在对等上下功夫, 还应力求译文之 “美”。必要时, 译文的意义和内容还可以有一定的发挥。歌德翻译《圣经》 的目的是要使德国老百姓都能读懂和接受《圣经》。正所谓 “言之无文, 行 而不远” , 文采必然成为他关注的一个主要问题, 因而也就必然从美学中汲 取灵感 [3]。

\section{2. 文学翻译思想}

17 到 18 世纪之间, 英国剧作家约翰・德莱顿拥护西塞罗的美学翻译观, 认为译者应重意不重词。他秉持一种中庸之道, 既反对过于呆板地遵循原文, 又反对过于随意的 “拟译” ——只把原文当作一个框架而在翻译中随意发挥。 德莱顿的翻译充分体现了这些原则; 蒲柏受新古典主义的影响, 译文用词考 究, 均匀工整, 但同时反对矫揉造作。德国思想家、作家歌德也认为要考虑 译文的可读性, 因而不必对原作亦步亦趋, 适当发挥创造性有利于异域文化 融入本土文化。以上三人的翻译观点源自自身文艺创作的实践, 而非纸上谈 兵, 因而具有较强的指导意义。此外, 近现代语文学派译论家、莎士比亚剧 作最杰出的德语译者施莱格尔、埃兹拉 - 庞德、艾 - 阿 - 瑞恰慈等人的翻译 理论也均彰显出哲学 - 美学倾向 [3] [4]。

\section{4. 近现代翻译思想}

19 世纪至今为现代译论期。19 世纪中晚期, 西方译论随历史比较语言学 兴起而由哲学 - 美学传统逐渐转向语言学视角, 翻译研究被视作语言学的分 支, 而到 60、70 年代又有将翻译研究作为独立学科的观点。这一时期的主要 特征是: 试图摆脱感性的、经验式的研究视角, 更加强调理性分析, 科学性、 系统性不断提升, 但有偏重字词、句子, 忽略话语、篇章的缺陷。

\section{1. 现代译论期的两个阶段}

现代译论期又分为两个阶段。第一阶段称为现代译论前期, 从 19 世纪末 到 20 世纪 50 年代, 恰好对应现代语言学前期。译论家雅可布森对诗歌翻译 的论述就基于对音位的分析。第一阶段中, 美学的影响有所减弱; 第二阶段 
从 20 世纪 60 年代直至现在, 为现代译论后期。这个时期译论仍从语言学中 汲取营养，但出现了一些新动向。首先，60 年代后卡特福德采用韩礼德基本 语言学理论研究双语转换。尤金 - 奈达则从结构语言学视角展开翻译研究, 其关键范畴是 “动态对等”。卡塔琳娜 - 赖斯、克里斯蒂安 - 诺德强调翻译 的目的性和文本的功能。新动向方面, 包括解构主义在内的后现代理论对西 方译论产生了巨大影响, 这在一定程度上显示了翻译研究由语言科学向文学 审美的回归。但 “后现代” 理论 “破而不立” 的局限性也同时显现出来。1990 年，苏珊 -巴斯内特和安德烈 - 勒菲弗尔编著的《翻译、历史与文化》标志 着翻译的文化转向。文化学派必然要关注文化研究, 而美学则是文化的重压 组成部分。以色列学者就提出翻译学不仅要突破语言学的限制, 也要突破文 学的限制, 向更广阔的文化领域迈进[3] [4]。

\section{2. 现代译论期著名翻译作品}

现代译论期涌现的著名译作有：英国语言学家麦克斯 - 缪勒借助比较语 言学翻译的《东方圣典丛书》，其中包括《梨俱吠陀》和《尚书》; 理雅各 采用语义翻译法翻译的《四书》、《五经》; 奈达本人虽未听说有什么译著 作，但他的翻译思想指导着整个联合圣经工会的翻译工作；葛浩文对莫言小 说的翻译体现了功能派和文化学派的思想。

\section{21世纪翻译思想}

到 21 世纪, 西方译论进入综合开发期, 积极吸收利用语言学、社会科学 以及自然科学研究成果 [4]。文学翻译始终是翻译领域举足轻重的一部分, 不 可能完全依赖纯理性的语言学。美学在今后的翻译研究中仍将发挥重要作用, 本文对译学美学渊源的梳理, 将为以此展开的翻译美学研究提供线索。在对 美学资源进行梳理的同时, 既要关注西方美学思想, 也要关注中国传统哲学 的美学思想，使两者共同为推进译论和整个翻译事业的发展做出贡献。对中 国传统美学思想与翻译关系的梳理至今为数不多，本文也试图起到抛砖引玉 的效果。

\section{Conflicts of Interest}

The authors declare no conflicts of interest.

\section{References}

[1] 王铁钧. 中国佛典翻译史稿[M]. 北京: 中央编译出版社, 2006 .

[2] 刘宓庆. 翻译美学导论 [M]. 北京: 中译出版社, 2019.

[3] 刘宓庆. 翻译与语言哲学 $[\mathrm{M}]$. 北京: 中译出版社, 2019.

[4] 杨建华. 西方译学理论辑要 [M]. 天津: 天津大学出版社, 2009. 


\section{Appendix (Abstract and Keywords in Chinese)}

\section{译学的美学渊源}

摘要：本文回顾了中西译论的发展历程。首先介绍了中国传统译论发展的三 个阶段，即从最初对 “信” 的重视到对 “文采” 的重视，再到后来的文质之 争。接着, 重点回顾了西方译论从古罗马时期到 20 世纪的发展史, 归纳出两 个主要发展时期, 即辞章美学时期和历史比较语言学时期。前一时期始于深 受柏拉图影响的西塞罗, 其思想影响了后来的《圣经》翻译和文学翻译; 后 一时期又分为两个阶段: 20 世纪 50 年代以前主要受结构主义语言学影响, 50 年代以后则深受新兴语言学派及现代思潮的影响。通过这些介绍, 呈现了中 西译论发展史的宏观框架。

关键词: 译论, 翻译史, 翻译观, 语言学 